\title{
UN MODELO DE PRONÓSTICO DE CONTAGIO
}

\section{Claudia I. Martínez García*}

Escuela Superior de Economía, Instituto Politécnico Nacional

\section{Adrián Hernández-del-Valle}

Escuela Superior de Economía, Instituto Politécnico Nacional

\section{Héctor Allier Campuzano}

Escuela Superior de Economía, Instituto Politécnico Nacional

(Recibido 3 de mayo 2004, aceptado 13 de agosto 2004)

\section{Resumen}

Construimos un modelo de vectores autorregresivos para pronosticar la volatilidad del Índice de Precios y Cotizaciones de la Bolsa Mexicana de Valores en función de otros portafolios de mercado, y probamos su poder predictivo ex-ante en el periodo enero 1997-diciembre 1998. Uno de los resultados más sorprendentes es que nuestro modelo hubiera permitido pronosticar los dos episodios de contagio que se presentaron en el intervalo de tiempo en cuestión: el efecto Dragón y el efecto Vodka.

\section{Abstract}

We build an autorregresive vectors model to forecast the volatility of the Índice de Precios y Cotizaciones of the Mexican Stock Exchange as a function of other market portfolios, and we test its ex-ante predictive power in the period January 1997-December 1998. One of the most surprising results is that our model would have been able to forecast the two contagion effects that ocurred in this time interval: The Dragon Effect and the Vodka Effect.

Clasificación JEL: C32, F30, F47, G15

Palabras clave: Contagio, Vectores Autorregresivos, Pronóstico

* Sección de Estudios de Posgrado e Investigación. Escuela Superior de Economía, IPN (SEPI-ESE-IPI). Manta 727, Col. Lindavista, C.P. 07300, México D.F. Tels. +52(55) 57142463. +52(55) 5752-2690. Correo electrónico: claimar27@hotmail.com y ahdv@hotmail.com 


\section{Introducción}

Contagio es que la crisis económica desatada en un país se transmita a otros. Su estudio es de gran importancia dados los efectos devastadores que genera. Por ejemplo, durante el Efecto Dragón -el episodio de contagio que empezó en Tailandia a mediados de 1997 y se propagó al mundo entero- la economía de Indonesia se contrajo 15\%, la de Malasia 7\%, Corea del Sur perdió 3\%, y Filipinas $4 \%$. La literatura de contagio gira en torno a dos grandes vertientes: la descripción de los episodios de contagio-el Efecto Dragón es especialmente popular- y la generación de herramientas de prevención. Este trabajo se enmarca en el segundo grupo. Construimos un modelo de vectores autorregresivos (VAR) para el pronóstico de la volatilidad del Índice de Precios y Cotizaciones $(I P y C)$ de la Bolsa Mexicana de Valores (BMV) bajo el supuesto de que una volatilidad superior a 0.05 persistente es indicio fuerte de contagio.

El VAR en cuestión es, hasta donde tenemos entendido, el primero de su tipo en el país, y probamos su poder predictivo ex-ante en el periodo 19971998 -el intervalo en cuestión estuvo marcado por dos episodios de infección significativos: el Efecto Dragón y el Efecto Vodka.

Los resultados más importantes son que el $\operatorname{VAR}(1,1)$ sí hubiera servido para predecir ambos contagios a casi un año de que ocurrieran; obtuvimos un residuo normal promedio de 0.00004 ; durante el contagio se observan volatilidades $>0.05$ de manera persistente; finalmente, la prueba de causalidad de Granger y las $R^{2}$ de modelos VAR, estimados con series de tiempo más largas, sugieren que en la actualidad se pueden construir modelos con un mayor poder predictivo, i.e. bastaría con observar la volatilidad del $I P y C$ para anticipar el contagio.

El VAR estimado es el siguiente:

$$
I P y C_{t+1}=0.445385 D J_{t}+0.151864 H S_{t-1}+0.145881 M E R_{t}+0.112298 I B O_{t}
$$

$$
+0.158967 N I K_{t-1}+0.233046 I P y C_{t-1} .
$$

Como se puede observar, la volatilidad del $I P$ yC , tomada como la desviación estándar de sus rendimientos diarios, resultó en función de las volatilidades del Dow Jones $(D J)$, del Hang Seng $(H S)$ de Hong Kong con un día de rezago, del Merval argentino ( $M E R$ ), del Indice Bovespa $(I B O)$ de la bolsa de Saõ Paulo, del Nikkei $(N I K)$ de Japón y del mismo IPyC, los dos últimos ambos con un rezago.

¿Por qué índices? y ¿por qué volatilidades? Se modelaron índices bursátiles por dos razones. Primero, porque en la práctica, uno de los efectos iniciales del contagio se observa en las bolsas, en particular, en sus portafolios de mercado. Por ejemplo, durante el Efecto Dragón, en Latinoamérica, la bolsa brasileña fue la primera en resentir los efectos. El Índice Bovespa acumuló una pérdida del $41 \%$ entre julio y noviembre de ese año; en el mismo lapso el Merval argentino perdió $27 \%$, mientras que el IPyC cayó $8 \%$ después de experimentar un ligero repunte. 
Figura 1. Merval, IPyC, Ibovespa durante el Efecto Dragón 1997.
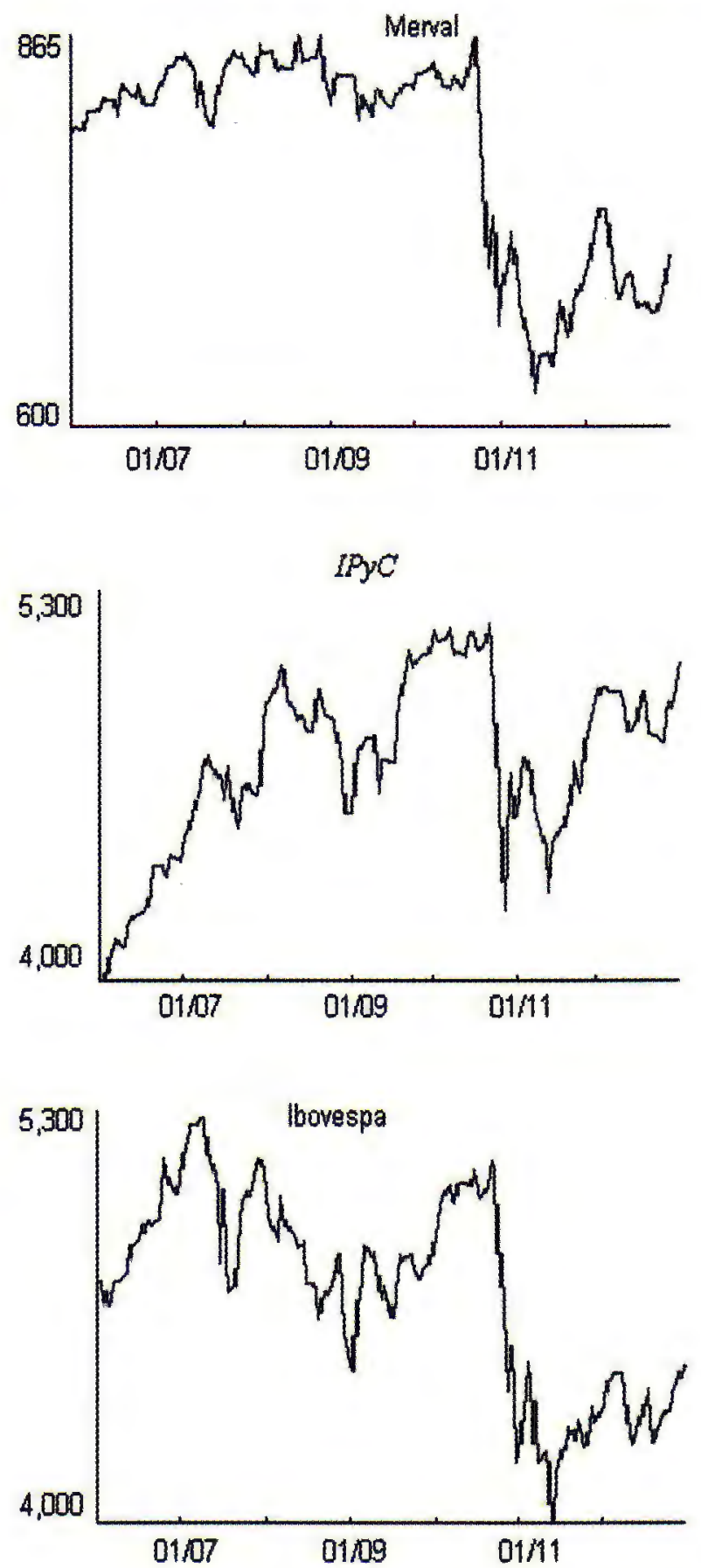
Segundo, la literatura que se avoca a la descripción del fenómeno de contagio se ha centrado en los canales mediante los cuales se transmiten las crisis. Un primer supracanal es la regionalidad -una manera geográfica de agrupar a los países- y condiconados a ésta se identifican dos tipos de canales de transmisión adicionales: los nexos de tipo comercial y los de naturaleza financiera (ver Eichengreen, Rose y Wyplosz (1996), Wolf (1997), Glick y Rose (1998), Calvo (1998), Kodres y Pritsker (1999), Bernard y Bisignano (2000)). Kaminsky y Reinhart (2000) estudian los diferentes canales, estiman probabilidades incondicionales y condicionales de contagio para cada uno, y concluyen que los canales financieros tienen una mayor incidencia en la infección tal como lo muestra la Tabla 1.

Tabla 1. Probabilidades condicionales de contagio por canal.

\begin{tabular}{|c|c|c|c|}
\hline & Canal de transmisión & $\mathrm{P}\left(C / X_{i}\right)$ & Promedio \\
\hline & $X_{1}$ Regionalidad & $57.06 \%$ & $57.06 \%$ \\
\hline $\begin{array}{c}\text { Nexos } \\
\text { financieros }\end{array}$ & $X_{2}$ fondos mutuos & $80.40 \%$ & $81.95 \%$ \\
\hline $\begin{array}{c}\text { Nexos } \\
\text { comerciales }\end{array}$ & $X_{3}$ acreedores bancarios comunes & $83.50 \%$ & \\
\hline
\end{tabular}

Como se puede observar, los canales de transmisión de tipo financiero, como el que modelamos en este trabajo, son en promedio el vehículo más fuerte del contagio, $81.95 \%$; le siguen los nexos comerciales, $57.50 \%$.

Figura 2. Ibovespa 1994-1998.

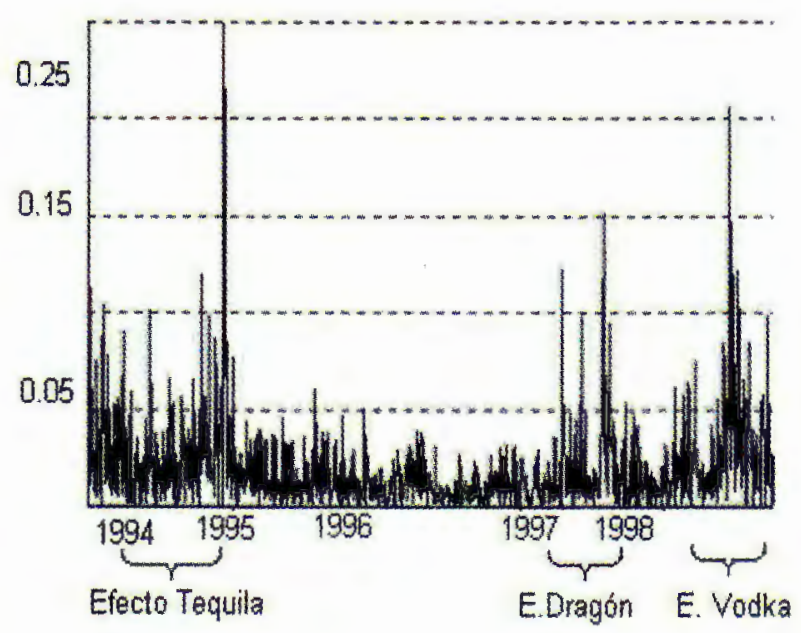


Por otra parte, se usaron series históricas de volatilidades diarias porque el contagio dispara la incertidumbre de los mercados, y ésta se refleja en una mayor desviación estándar de los rendimientos de los precios -la volatilidad. En la Figura 2 se puede observar el comportamiento de la volatilidad del Índice Bovespa entre 1994 y 1998, que abarca los efectos Tequila, Dragón y Vodka.

Finalmente, para poder probar el poder predictivo ex-ante del modelo, el $\operatorname{VAR}(1,1)$ se estimó para el periodo 5/12/1994-31/12/1996, extrapolamos 19971998, y comparamos los resultados con la información 1/1/1997-31/12/1998 que se tomó fuera de muestra. La Figura 3 compara la volatilidad extrapolada con base en el modelo, contra la observada en el intervalo de tiempo citado.

Figura 3. Volatilidad del IPyC 1997-1998.

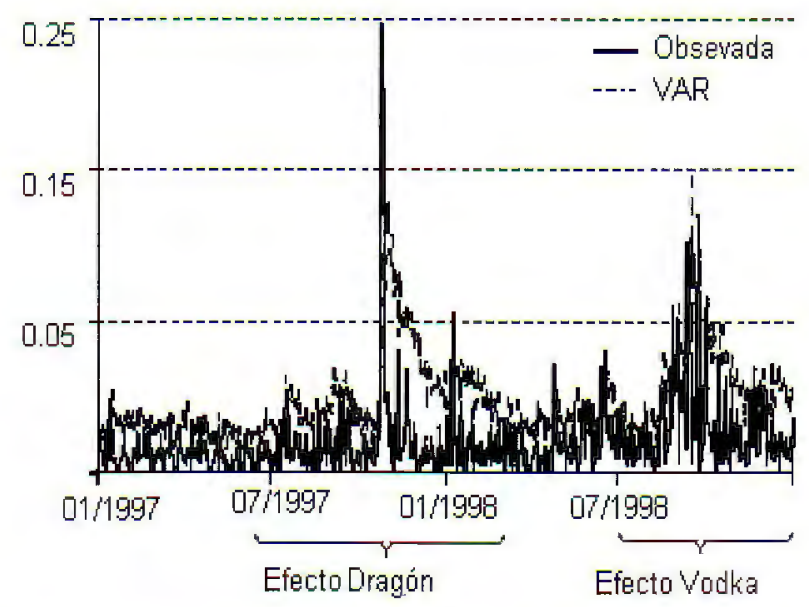

En la sección 2 reportamos la metodología que seguimos para la identificación de los focos de contagio. En la sección 3 presentamos la especificación del modelo y los resultados observados. Finalmente, en la sección 4 incluimos algunas conclusiones y posibles líneas de investigación.

\section{Focos de contagio}

Como se mencionó con anterioridad, los canales a través de los cuales se da el contagio han sido el punto principal de atención de los diversos autores dedicados a la investigación de este fenómeno.

Para la identificación de posibles países foco de contagio para México, partimos de la investigación realizada por Kaminsky y Reinhart (2000) por dos razones: primero, porque analizan y describen tanto los canales derivados del comercio como los derivados de los mercados financieros. La mayoría de los investigadores tienden a concentrarse en un solo canal; y segundo, porque cuantifican la importancia de cada canal en el proceso de contagio mediante el cálculo de probabilidades condicionales para cada uno de los canales - Tabla 1.

Kaminsky y Reinhart (2000) agrupan los canales de transmisión en tres rubros. El primero denominado nexos financieros incluye dos canales de transmisión: los acreedores bancarios comunes y los fondos mutuos; el segundo, 
denominado nexos comerciales comprende otros dos canales: el comercio bilateral y el comercio con un tercer mercado común. La regionalidad subyace a los demás.

A continuación se describen cada uno de los canales de transmisión, y se especifica el proceso que se llevó a cabo para el rastreo de países que podrían tener una incidencia sobre México en el evento de un contagio.

\subsection{Regionalidad}

La regionalidad se refiere al hecho de que el contagio es más factible si existe cercanía geográfica. Kaminsky y Reinhart (2000) calculan dos probabilidades de contagio:

1. La incondicional $P(C)$ : la probabilidad de que exista crisis en un país sin que exista en otro(s); y

2. La condicional $P(C \mid E)$ : la probabilidad de contagio dado que existe crisis en otro(s) país(es) de la misma región.

En la Tabla 2 se puede observar que la probabilidad condicional de contagio es directamente proporcional a la cantidad de países infectados en una región. Por ejemplo, en Latinoamérica se observa que la probabilidad de que una economía se infecte pasa del $29.4 \%$ al $68.8 \%$ cuando el número de países enfermos en la región se incrementa del $25 \%$ al $51 \%$. La relación se sostiene en las otras regiones muestreadas.

Tabla 2. La incidencia y evolución del contagio regional: Asia, Europa y Latinoamérica.

\begin{tabular}{ccccccc}
\hline $\begin{array}{l}\text { Proporción } \\
\text { de los países } \\
\text { en la región } \\
\text { con crisis(\%) }\end{array}$ & & Asia & \multicolumn{2}{c}{ Europa } & \multicolumn{2}{c}{ Latinoamérica } \\
\hline & $P(C)$ & $P(C \mid E)$ & $P(C)$ & $P(C \mid E)$ & $P(C)$ & $P(C \mid E)$ \\
$0-25$ & 26.8 & 19.8 & 28.6 & 14.7 & 29.4 & 18.3 \\
$25-50$ & 26.8 & 15.3 & 28.6 & 32.3 & 29.4 & 30.8 \\
50 ó más & 26.8 & 67.4 & 28.6 & 35.0 & 29.4 & 68.8 \\
\hline
\end{tabular}

En el caso de México, además de las principales economías latinoaméricanas (Argentina, Brasil, Colombia, Chile y Venezuela) se incluyó a Estados Unidos por su cercanía y peso natural.

\subsection{Nexos financieros: acreedores bancarios comunes}

Una economía tiene, en general, dos fuentes de financiamiento externo o nexos financieros: insitituciones bancarias y fondos mutuos. Los flujos de estos agentes son altamente procíclicos, Ocampo (2002) señala que existe una correlación fuerte entre los periodos de expansión (contracción) de las economías emergentes y la entrada (salida) de capitales. En esta sección abordamos a los primeros.

El canal de acreedores bancarios se refiere al hecho de que existen bloques de países que tienen a otro como acreedor bancario común. En este caso la 
expansión de las crisis tiene la lógica siguiente: si un banco se enfrenta al incremento de la cartera vencida otorgada a un país, se verá obligado a reducir su exposición al riesgo abandonando otros proyectos que puedan colocarlo en una posición más vulnerable, y contraerá el crédito pues se verá forzado a recapitalizar, prever y ajustar la baja en su nivel de riqueza. Esta contracción afectará a países en la misma región -o con el mismo nivel de riesgo, e.g. emergentes- y tendrá una incidencia negativa sobre su crecimiento.

Por ejemplo, supóngase que Argentina y México tienen el mismo nivel de riesgo país y que tienen a Estados Unidos como acreedor bancario común. Si Argentina entra en moratoria, el argumento anterior anticiparía que los bancos norteamericanos frenarán sus créditos a Argentina y a México, y esto frenará el crecimiento de ambos.

Kaminsky y Reinhart (2000) calculan las probabilidades correspondientes con base en una muestra mensual de 1970 a 1998 , que incluye 80 episodios de crisis monetarias de distintos países tanto industrializados como en desarrollo. Forman dos subgrupos con los países pertenecientes a su muestra. Uno formado por los países cuya deuda está mayormente contratada con Estados Unidos: Brasil, Argentina, Chile, Colombia, México, Filipinas, Uruguay y Venezuela; y otro en el que está mayormente contratada con Japón: Indonesia, Malasia y Tailandia. Los resultados son los siguientes:

Tabla 3. Contagio y acreedores bancarios comunes.

\begin{tabular}{ccc}
\hline $\begin{array}{c}\text { Proporción de los países } \\
\text { en la región con crisis }(\%)\end{array}$ & \\
\hline & $P(C)$ & $P(C \mid E)$ \\
$0-25$ & 31.5 & 19.2 \\
$25-50$ & 31.5 & 28.4 \\
50 ó más & 31.5 & 83.5 \\
\hline
\end{tabular}

La Tabla 3 se interpreta de la manera siguiente: la probabilidad de que un país entre en crisis cuando el $51 \%$ de los países geográficamente cercanos a él sufren una crisis y además comparten un mismo acreedor bancario es del $83.5 \%$.

En lo que respecta a México, el Banco Internacional de Acuerdos (Bank of International Settlements) proporciona estadísticas respecto a acreedores bancarios como porcentaje de la deuda total de un país. La Tabla 4 muestra un resumen de las economías que guardaban una relación de regionalidad y de acreedores bancarios con México en 1997.

Tabla 4. Pasivos como porcentaje de la deuda total (\%).

\begin{tabular}{|c|c|c|c|c|}
\hline Composición de pasivos & Francia & Alemania & Japón & Holanda \\
\hline Argentina & 7.91 & 16.90 & 4.40 & 5.56 \\
\hline Brasil & 9.00 & 14.62 & 7.78 & 7.89 \\
\hline Chile & 5.64 & 17.04 & 5.52 & 9.60 \\
\hline Colombia & 8.87 & 13.56 & 12.60 & 6.04 \\
\hline México & 11.36 & 8.23 & 8.00 & 4.87 \\
\hline Venezuela & 11.38 & 15.71 & 4.87 & 4.67 \\
\hline
\end{tabular}


Tabla 4 (continuación).

\begin{tabular}{|c|c|c|c|c|}
\hline Composición de pasivos & España & Reino Unido & EE.UU & Otros \\
\hline Argentina & 4.73 & 5.51 & 28.97 & 26.02 \\
\hline Brasil & 3.21 & 6.09 & 25.21 & 26.20 \\
\hline Chile & 10.40 & 3.80 & 28.28 & 19.72 \\
\hline Colombia & 7.69 & 8.58 & 24.68 & 17.98 \\
\hline México & 5.18 & 7.42 & 29.45 & 25.49 \\
\hline Venezuela & 3.17 & 11.44 & 25.85 & 22.91 \\
\hline
\end{tabular}

\subsection{Nexos financieros: canales de liquidez y fondos mutuos}

La segunda fuente de financiamiento externo de una economía la constituyen los fondos mutuos que surgieron a mediados de los años noventa. Su incidencia en los episodios de contagio sigue una lógica similar a la de los acreedores bancarios.

En general, el problema radica en la liquidez. Por una parte, un mercado más líquido o bursátil - uno del que se puede entrar o salir con mayor facilidades más atractivo para el inversionista. Por la otra, la amplitud y profundidad de los mercados financieros proporcionan opciones importantes de financiamiento para las empresas. Es decir, un mercado líquido es deseable. Sin embargo, esta misma característica incrementa la volatilidad de los flujos, en última instancia, ell caso de nerviosismo o crisis, pueden desaparecer con sólo apretar un botón.

Kaminsky y Reinhart (2000) argumentan que este canal está relacionado con la interdependencia entre mercados accionarios. Con base en este argumento, calculan la correlación entre los respectivos portafolios de mercado, y después establecen una clasificación:

- correlación baja: entre 0 y 0.20 ,

- correlación moderada: entre 0.21 y 0.40, y

- correlación alta: > 0.40.

Con los resultados anteriores estiman probabilidades condicionales e incondicionales de contagio. La Tabla 5 reporta sus resultados.

Tabla 5. Contagio y mercados altamente correlacionados.

\begin{tabular}{ccc}
\hline $\begin{array}{c}\text { Proporción de los países } \\
\text { en la. región con crisis (\%) }\end{array}$ & & \\
\hline & $P(C)$ & $P(C \mid E)$ \\
$0-25$ & 33.3 & 5.5 \\
$25-50$ & 33.3 & 54.1 \\
50 ó más & 33.3 & 80.4 \\
\hline
\end{tabular}

Es decir, la probabilidad de que un país se contagie cuando el $51 \%$ de los países de la región están enfermos y existe una alta correlación entre sus mercados bursátiles es de $80.4 \%$. Los resultados del ejercicio anterior aplicado a México se muestran en la Tabla 6. 
Tabla 6. Correlaciones de los mercados financieros con México.

\begin{tabular}{|c|c|c|c|c|c|}
\hline País & México & EE.UU. & Argentina & Brasil & Venezuela \\
\hline México & 1 & 0.90 & 0.93 & 0.91 & 0.19 \\
\hline EE.UU. & 0.90 & 1.00 & 0.82 & 0.88 & 0.58 \\
\hline Argentina & 0.93 & 0.82 & 1.00 & 0.89 & 0.44 \\
\hline Brasil & 0.91 & 0.88 & 0.89 & 1.00 & 0.55 \\
\hline Venezuela & 0.19 & 0.58 & 0.44 & 0.55 & 1.00 \\
\hline Colombia & -0.22 & -0.77 & -0.74 & -0.86 & -0.47 \\
\hline China-HongKong & 0.87 & 0.97 & 0.82 & 0.88 & 0.64 \\
\hline Japón & 0.85 & 0.71 & 0.81 & 0.78 & 0.10 \\
\hline Chile & 0.34 & 0.45 & $\ldots$ & $\ldots$ & 0.01 \\
\hline
\end{tabular}

\begin{tabular}{|c|c|c|c|c|}
\hline País & Colombia & China-Hong Kong & Japón & Chile \\
\hline México & -0.22 & 0.87 & 0.85 & 0.34 \\
\hline EE.UU. & -0.77 & 0.97 & 0.71 & 0.45 \\
\hline Argentina & -0.74 & 0.82 & 0.81 & $\ldots$ \\
\hline Brasil & -0.86 & 0.88 & 0.78 & $\ldots$ \\
\hline Venezuela & 0.24 & 0.64 & 0.19 & 0.01 \\
\hline Colombia & 1.00 & -0.77 & -0.73 & 0.02 \\
\hline China-HongKong & -0.77 & 1.00 & 0.66 & 0.07 \\
\hline Japón & -0.73 & 0.66 & 1.00 & $\ldots$ \\
\hline Chile & 0.02 & 0.07 & $\ldots$ & 1.00 \\
\hline
\end{tabular}

Los posibles focos de contagio para México, con base en el criterio establecido y la Tabla 6 son: Estados Unidos, Argentina, Brasil, Japón y China-Hong Kong.

\subsection{Nexos comerciales: comercio bilateral}

Los canales de transmisión incluidos en este rubro están relacionados con el comercio de bienes y servicios entre países. El comercio bilateral en particular se refiere a la relación entre dos de ellos, y la probabilidad de contagio se da cuando uno de los dos países entra en crisis. Las probabilidades de contagio incondicional y condicional que encontraron Kaminsky y Reinhart respecto al comercio bilateral son:

Tabla 7. Contagio y comercio bilateral.

\begin{tabular}{ccc}
\hline $\begin{array}{c}\text { Proporción de los países en } \\
\text { la región con crizis (\%) }\end{array}$ & & \\
\hline & $P(C)$ & $P(C \mid E)$ \\
$0-25$ & 37.4 & 29.3 \\
$25-50$ & 37.4 & 15.6 \\
50 ó más & 37.4 & 84.7 \\
\hline
\end{tabular}


Los resultados indican que la probabilidad de que un país se contagie cuando el $51 \%$ de los países de la región presentan problemas de crisis y existe comercio bilateral entre ellos y el país en cuestión es del $84.7 \%$.

El mayor socio comercial de México es Estados Unidos, mientras que México es el segundo socio comercial de los Estados Unidos detrás de Canadá y por encima de Japón.

\subsection{Nexos comerciales: comercio con un tercer mercado común}

El comercio con terceros se refiere a la existencia de un mercado común en el que compiten un grupo de países. Las probabilidades indican que este canal no es significativo en el proceso de contagio tal como lo muestra la Figura 11.

Tabla 8. Contagio y nexos comerciales.

\begin{tabular}{ccc}
\hline $\begin{array}{c}\text { Proporción de los países en } \\
\text { la región con crisis (\%) }\end{array}$ & & \\
\hline & $P(C)$ & $P(C \mid E)$ \\
$0-25$ & 27.6 & 5.8 \\
$25-50$ & 27.6 & 6.3 \\
50 ó más & 27.6 & 30.7 \\
\hline
\end{tabular}

\subsection{Resumen de focos de contagio}

Con base en lo descrito en esta sección se asevera que los posibles focos de contagio para México son: Argentina, Brasil, Estados Unidos, China-Hong Kong y Japón. La Tabla 9 resume los resultados.

Tabla 9. Resumen de focos de contagio con base en los canales de transmisión.

\begin{tabular}{|c|c|c|c|c|c|}
\hline País & Regionalidad & $\begin{array}{c}\text { Acreedroes } \\
\text { bancarios } \\
\text { comunes }\end{array}$ & $\begin{array}{c}\text { Fondos } \\
\text { mutuos }\end{array}$ & $\begin{array}{c}\text { Comercio } \\
\text { Bilateral }\end{array}$ & $\begin{array}{c}\text { Comercio } \\
\text { con } \\
\text { terceros }\end{array}$ \\
\hline Argentina & $\bullet$ & $\bullet$ & $\bullet$ & & \\
\hline Brasil & $\bullet$ & $\bullet$ & $\bullet$ & & \\
\hline Colombia & $\bullet$ & $\bullet$ & & & \\
\hline Chile & $\bullet$ & $\bullet$ & & & \\
\hline China-Hong Kong & & & $\bullet$ & & \\
\hline Estados Unidos & $\bullet$ & & $\bullet$ & $\bullet$ & \\
\hline Japón & & & $\bullet$ & & \\
\hline Venezuela & $\bullet$ & $\bullet$ & & & \\
\hline
\end{tabular}

Se excluyeron Colombia, Chile y Venezuela por el tamaño de sus mercados accionarios en el periodo de estudio 1994-1998. En la sección siguiente se especifica el procedimiento que se siguió para modelar el VAR $(1,1)$ y los resultados. 


\section{El modelo de pronóstico de contagio para México}

Una vez identificados Estados Unidos, Brasil, Argentina, Japón y China-Hong Kong como focos de contagio, construimos series de tiempo de volatilidades diarias de sus portafolios de mercado: Dow Jones y Nasdaq (E.U.A.), Índice Bovespa (Brasil), Merval (Argentina), Nikkei (Japón) y Hang Seng (ChinaHong Kong). Además incluímos el FTSE 100 de Inglaterra, el CAC40 parisino y el DAX de la bolsa de Frankfurt para probar la hipótesis de que ningún país europeo es foco de contagio. La Tabla 10 presenta los resultados de la prueba de causalidad de Granger con hipótesis nula:

$$
H_{0}:=X_{i} \quad \text { no condiciona } \quad X_{j}
$$

Tabla 10. Prueba de causalidad de Granger con 4 rezagos.

\begin{tabular}{|c|c|c|c|}
\hline Hipótesis Nula: & Obs & F-Estadístico & Probabilidad \\
\hline$D J$ no Granger condiciona $I P y C$ & 540 & 1.342360 & 0.25300 \\
$I P y C$ no Granger condiciona $D J$ & & 0.81336 & 0.51695 \\
\hline$C A C 40$ no Granger condiciona $I P y C$ & 540 & 0.95660 & 0.43093 \\
$I P y C$ no Granger condiciona $C A C 40$ & & 2.29095 & 0.05858 \\
\hline$D$ AX no Granger condiciona $I P y C$ & 540 & 0.182260 & 0.94759 \\
$I P y C$ no Granger condiciona $D A X$ & & 0.79905 & 0.52612 \\
\hline$F T S E 100$ no Granger condiciona $I P y C$ & 540 & 0.43633 & 0.78239 \\
$I P y C$ no Granger condciona $F T S E 100$ & & 1.39066 & 0.23588 \\
\hline$H S$ no Granger condiciona $I P y C$ & 540 & 4.56692 & 0.00124 \\
$I P y C$ no Granger condciona $H S$ & & 3.61972 & 0.23588 \\
\hline$I B O$ no Granger condiciona $I P y C$ & 540 & 4.32003 & 0.00190 \\
$I P y C$ no Granger condciona $I B O$ & & 3.83452 & 0.00440 \\
\hline$M E R$ no Granger condiciona $I P y C$ & 540 & 2.75022 & 0.02761 \\
$I P y C$ no Granger condciona $M E R$ & & 2.38787 & 0.05007 \\
\hline$N A S D A Q$ no Granger condiciona $I P y C$ & 540 & 1.93824 & 0.10272 \\
$I P y C$ no Granger condciona $N A S D A Q$ & & 0.25803 & 0.90474 \\
\hline$N I K$ no Granger condiciona $I P y C$ & 540 & 3.80257 & 0.00465 \\
$I P y C$ no Granger Condciona $N I K$ & & 1.94216 & 0.10211 \\
\hline
\end{tabular}

Como se puede observar, la prueba de Granger corrobora los resultados de la identificación de focos de contagio: ningún índice europeo incide sobre el IPyC; mientras que China-Hong Kong, Brasil, el Merval y el Nikkei sí. El resultado más sorprendente es que ningún índice norteamericano muestra una relación de causalidad con el $I P y C$.

En busca de una mayor certidumbre en particular respecto al Dow Jones y Nasdaq, hicimos una primera estimación VAR con todos los índices citados, y en la corrección del modelo desechamos aquellos cuyos parámetros resultaron estadísticamente no significativos, i.e. aquellos con un t-estadístico $<|2|$. La Tabla 11 muestra esta primera estimación. 
Tabla 11. Primera estimación. Muestra: 5/12/1994 - 31/12/1996.

Número de observaciones: 542 . t-estadístico entre paréntesis.

\begin{tabular}{|c|c|}
\hline$I P y C(-1)$ & $\begin{array}{l}0.2114557 \\
(5.09618)\end{array}$ \\
\hline$N I K(-1)$ & $\begin{array}{l}0.149219 \\
(2.31816)\end{array}$ \\
\hline$H K(-1)$ & $\begin{array}{l}0.155523 \\
2.31518)\end{array}$ \\
\hline$D J$ & $\begin{array}{l}0.368129 \\
(2.58593)\end{array}$ \\
\hline$D J(-1)$ & $\begin{array}{c}-0.16677 \\
(-1.12437)\end{array}$ \\
\hline$N A S D A Q$ & $\begin{array}{l}0.172462 \\
(1.56807)\end{array}$ \\
\hline$N A S D A Q(-1)$ & $\begin{array}{c}-0.00954 \\
(-0.08839)\end{array}$ \\
\hline$M E R$ & $\begin{array}{c}0.12985 \\
(3.29049)\end{array}$ \\
\hline$M E R(-1)$ & $\begin{array}{c}0.03351 \\
(0.82868)\end{array}$ \\
\hline$I B O$ & $\begin{array}{l}0.097423 \\
(3.13604)\end{array}$ \\
\hline$I B O(-1)$ & $\begin{array}{c}0.023065 \\
(0.74842)\end{array}$ \\
\hline FTSE 100 & $\begin{array}{c}0.28742 \\
(1.89298)\end{array}$ \\
\hline FTSE $100(-1)$ & $\begin{array}{l}0.073569 \\
(0.48528)\end{array}$ \\
\hline$C A C 40$ & $\begin{array}{l}0.090949 \\
(0.97784)\end{array}$ \\
\hline$C A C 40(-1)$ & $\begin{array}{l}0.051545 \\
(0.55108)\end{array}$ \\
\hline$D A X$ & $\begin{array}{c}0.191738 \\
(1.83245)\end{array}$ \\
\hline$D A X(-1)$ & $\begin{array}{c}-0.17983 \\
(-1.74968) \\
\end{array}$ \\
\hline $\begin{array}{c}\text { R-squared } \\
\text { Adj. R-squared }\end{array}$ & $\begin{array}{l}0.253486 \\
0.230736\end{array}$ \\
\hline
\end{tabular}

El modelo corregido se muestra en la Tabla 12. 
Tabla 12: Modelo corregido. Muestra: 5/12/1994 - 31/12/1996.

Número de observaciones: 542 . t-estadístico entre paréntesis.

\begin{tabular}{cc}
\hline$I P_{y} C(-1)$ & 0.233046 \\
& $(6.09042)$ \\
$N I K(-1)$ & 0.158967 \\
& $(2.73928)$ \\
$H S(-1)$ & 0.151864 \\
& $(2.44027)$ \\
$D J$ & 0.445385 \\
& $(4.18201)$ \\
$M E R$ & 0.145881 \\
& $(3.88652)$ \\
$I B O$ & 0.112298 \\
& $(3.90925)$ \\
\hline R-squared & 0.235761 \\
Adj. R-squared & 0.228632 \\
\hline
\end{tabular}

Una vez estimado el VAR para la volatilidad del IP $y C$, nos enfrentamos a la dificultad de que para extrapolarlo en el intervalo de tiempo citado teníamos dos opciones:

1. Tomar los datos observados de la volatilidad de cada índice, o

2. Modelar la volatilidad de cada índice en el periodo 5/12/1994-31/12/1996; tomar el intervalo 1/0/1997-31/12/1998 fuera de muestra; extrapolar cada índice en el intervalo fuera de muestra; y usar estos datos en el VAR.

Optamos por la segunda en un intento por realizar una verdadera prueba del poder predictivo ex-ante de nuestro modelo, i.e. lo más próximo posible a una circunstancia real. La volatilidad de cada índice se estimó usando modelación ARMA-GARCH. La Tabla 13 reporta los resultados.

Tabla 13. Modelos ARMA de volatilidades.

\begin{tabular}{|c|c|c|c|c|c|}
\hline Índice & Hang Seng & Merval & Bovespa & Nikkei & Dow Jones \\
\hline $\mathrm{AR}(1)$ & 1.243995 & 0.996844 & 1.0000096 & 1.322321 & 0.076742 \\
\hline $\mathrm{AR}(2)$ & -0.246662 & & & -0.3227560 & 0.912790 \\
\hline $\mathrm{MA}(1)$ & -0.968148 & -0.873749 & -0.911150 & -0.972594 & \\
\hline $\mathrm{MA}(2)$ & & & & & -0.881737 \\
\hline
\end{tabular}

Finalmente, extrapolando la volatilidad de cada portafolio de mercado y luego la volatilidad del $I P y C$ con base en el VAR $(1,1)$, se obtuvieron los siguientes resultados:

- A manera de referencia, la mayor volatilidad en el periodo se observó el 24 de octubre de 1997, cuando la desviación estándar del IPyC alcanzó 0.176960. 
- Durante los dos episodios de contagio observados en el intervalo de interés se obtuvo una desviación estándar $>0.05$.

- El residuo promedio del VAR $(1,1)$ fue 0.028753 , con una desviación estándar de 0.016725 .

- La serie de residuos de las volatilidades observada y estimada normalizadas arrojó un error promedio de 0.00004 con desviación estándar de 0.28285 .

La Figura 4 en la introducción compara el VAR (1.1) con la volatilidad observada del $I P y C$ en el periodo fuera de muestra. La Figura 4 compara ambas series normalizadas.

Figura 4. Volatilidades normalizadas.

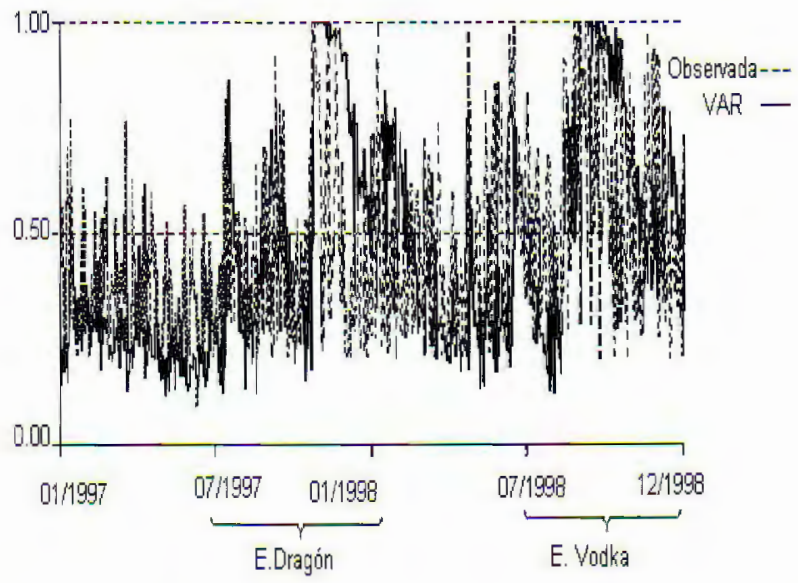

\section{Conclusiones}

Recapitulando, el procedimiento que se siguió para la construcción del modelo es el siguiente:

1. Identificar los focos de contagio,

2. Construir series de desviaciones estándar diarias de los portafolios de mercado de cada país identificado como foco de contagio,

3. Modelar la volatilidad del índice dependiente en función de los independientes mediante Vectores Autorregresivos,

4. Modelar cada índice independiente usando ARMA-GARCH y extrapolarlo,

5. Extrapolar la volatilidad del índice dependiente con base en los pasos anteriores.

Las Figuras 3 y 4 sugieren que nuestro modelo hubiera sido capaz de anticipar los Efectos Dragón y Vodka a casi un año de que sucedieran.

Conviene mencionar que la regionalidad parece haber cedido su lugar al ríesgo país, o que las regiones han cambiado. Por ejemplo, México no se enfermó ante la crisis Argentina de 2002. Lo anterior se puede explicar por el hecho de 
que México tenía un riesgo país muy distinto al de Argentina: alrededor de 200 puntos base para México y cerca de 7,000 para Argentina, o porque a México lo agrupan con EE.UU y Canadá.

Por último, se resaltan dos líneas de investigación claras. Primero, pruebas de Granger usando series de volatilidades en el intervalo 1994-2001 arrojan causalidades más fuertes entre los portafolios de mercado, esto se puede explicar por la convergencia de las economías, e implica que modelos actuales similares al nuestro tendrían un mayor poder predictivo ex-ante.

Segundo se puede construir un Mapa de Contagio, e.g. para México se encontró que el Dow Jones, el Hang Seng, el Nikkei, el Bovespa y el Merval inciden en el comportamiento de la volatilidad del IPyC. Usando el mismo procedimiento descrito en este trabajo, se identifican los índices que condicionan a estos portafolios de mercado, y luego a los que condicionan a aquellos, tantas veces como sea deseable o necesario. Al final se tendría una especie de red, de tal forma que cuando surja una crisis económica en un país, se podrá rastrear el efecto que tendrá sobre el $I P y C$. La Figura 5 resume esta idea:

Figura 5. Mapa de Contagio.

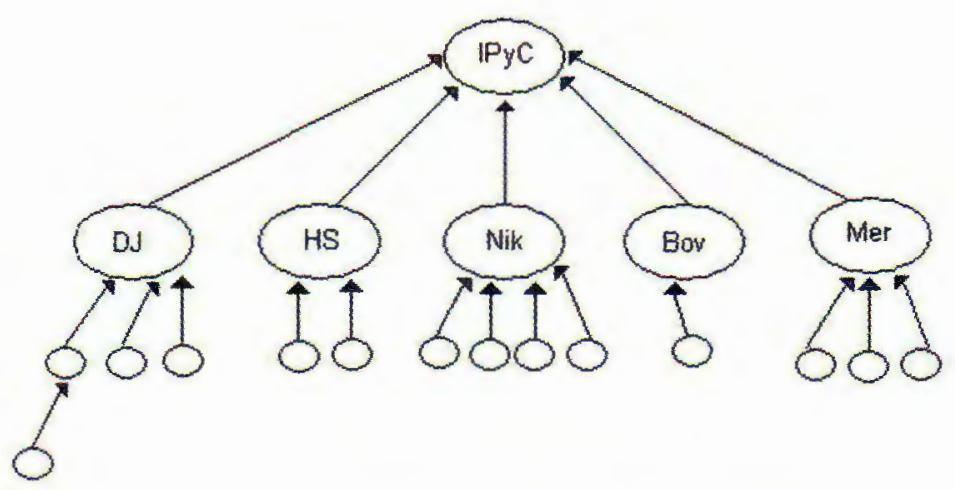

\section{Bibliografía}

Bernard, H. and J. Bisignano (2000). Information, Liquidity and Risk in the International Interbank Market: Implicit Guarantees and Private Credit Market Failure. Bank of International Settlements. Monetary and Economic Department.

Calvo, G. G. (1998). Capital Market Contagion and Recession: an Explanation of the Russian Virus. University of Maryland.

Eichengreen, B., A. Rose, and C. Wyplosz (1996). Contagious Currency Crises. Working Paper. National Bureau of Economic Research. No. 5681.

Glick, R. and A. Rose (1998). Currency and Banking Crises: The early Warnings of Distress. International Finance. Discussion Paper. No. 629. Board of Governors of the Federal Reserve System.

Kaminsky, G. and C. M. Reinhart (2000). On Crises, Contagion, and Confusion. Journal of International Economics, 51, pp. 145-168.

Kodres, L. E. and M. Pritsker (1999). A Rational Expectations Model of Financial Contagion. International Monetary Fund. Washington D.C.

Ocampo, J. A. (2002). Developing Countries Anti-cyclical Policies in a Globalized World. Santiago. CEPAL.

Wolf, H. C. (1997). Regional Contagion Effects in Emerging Markets. Working Papers in International Economics. Vol. G-97-03. Princeton University. 